\title{
Schistosomiasis in Iran, From the Past Till Elimination
}

\author{
Seyed Mohammad Alavi, ${ }^{1, *}$ and Shokrollah Salmanzadeh ${ }^{2}$ \\ ${ }^{1}$ Health Research Institute, Infectious and Tropical Diseases Research Center, Ahvaz Jundishapur University of Medical Sciences, Ahvaz, IR Iran \\ ${ }^{2}$ Department of Infectious Diseases, Razi Hospital, Ahvaz Jundishapur University of Medical Sciences, Ahvaz, IR Iran \\ "Corresponding author: Seyed Mohammad Alavi, Health Research Institute, Infectious and Tropical Diseases Research Center, Ahvaz Jundishapur University of Medical \\ Sciences, Ahvaz, IR Iran. Tel: +98-6133387724, Fax: +98-6113335396, E-mail: aseyedmohammad119@yahoo.com; alavi-sm@ajuma.ac.ir
}

Received 2016 January 15; Accepted 2016 January 19.

\begin{abstract}
Context: Schistosomiasis is a waterborne parasitic disease and the second most prevalent tropical disease after malaria infection. World health organization (WHO) considers it as a neglected tropical disease. Here, we aimed to research the epidemiology of schistosomiasis in Iran from the past to the elimination time.

Evidence Acquisition: For this study, we searched medical databases (PubMed, ISI, Scopus, Iran Medex, and Magiran) from January 1949 to the end of 2012. Keywords were epidemiology, schistosomiasis, Iran, and parasitic infection.

Results: On the basis of our results, using a national program for schistosomiasis control based on active and passive case finding, snail control programs, education of people, conducting training courses for health workers, and improvement of water supply for people living in endemic areas, the infection is now eliminated from Iran.

Conclusions: Schistosomiasis is one of the major tropical and communicable diseases in the eastern Mediterranean region (EMR). However, today it is eliminated in Iran by schistosomiasis control practices.
\end{abstract}

Keywords: Epidemiology, Iran, Parasitic Infection, Schistosomiasis

\section{Context}

Schistosomiasis (also known as bilharzia), a waterborne parasitic disease, is the second most prevalent tropical disease after malaria infection. World health organization (WHO) considers it as a neglected tropical disease. Schistosomiasis is endemic in 74 developing countries worldwide, infecting more than 200 million people in rural and peri-urban areas. Of these, 120 million have symptoms of the disease, of which 20 million have severe consequences. It is estimated that 650 million people worldwide live in endemic areas and are at risk of acquiring the infection (1-6). Schistosomiasis represents one of the major communicable diseases of public health and has socio-economic importance in the eastern Mediterranean region (EMR). The Schistosoma species that exist in EMR countries are the two human schistosomes, Schistosoma mansoni and S. haematobium. S. mansoni exist in eight EMR countries (Libya, Egypt, Saudi Arabia, Oman, Sudan, Yemen, Djibouti and Somalia) and S. haematobium exists in 14 EMR countries (Libya, Egypt, Saudi Arabia, Sudan, Yemen, Djibouti, Somalia, Jordan, Morocco, Tunisia, Lebanon, Syria, Iraq and Iran) (7). In recent years, WHO has reported a notable decrease in the prevalence and morbidity of the disease in many of these endemic countries. Today, in some of these countries, urinary schistosomiasis infection has been eliminated.

\section{Evidence Acquisition}

We searched medical databases (PubMed, ISI, Scopus, Iran Medex and Magiran) from January 1949 to the end of 2012. Keywords were epidemiology, schistosomiasis, Iran, and parasitic infection.

\section{Results}

We found many papers on the transmission of the infection, treatment and preventive methods; however, we focused on the burden of disease in Iran and the strategy for elimination phase.

\subsection{Schistosomiasis in Iran}

In Iran, Khuzestan is the only province in which schistosomiasis has been reported. Khuzestan is located in southwest of Iran and can be basically divided into two regions, the rolling hills and mountainous regions located in north of the Ahvaz ridge, and the plains and marsh lands to its south. The province covers an area of about $157000 \mathrm{~km}^{2}$ and has a population of approximately 4510127 (3177303 in urban and 1332824 in rural areas). It is a continuation of the Mesopotamian plain, and is bordered on the south by Persian Gulf, on the west by Iraq, and on the north and east by Zagros mountains. The area is irrigated by Karoun, 
Karkheh, Jarahi and Maroun rivers (8). Khuzestan has great potentials for agricultural expansion, which is almost unrivaled by the country's other provinces. Large and permanent rivers flow over the entire territory, contributing to the fertility of the land. Karun, Iran's most effluent river, 850 kilometers long, flows into the Persian Gulf through this province. The climate of Khuzestan is generally hot and occasionally humid, particularly in the south, while winters are much more pleasant and dry. Summer time temperature routinely exceeds $50^{\circ} \mathrm{C}$ and in winter it can drop below freezing, with occasional snowfall, all the way south to Ahvaz. Khuzestan is known to master the hottest temperatures on record for a populated city anywhere in the world. Many sandstorms and dust storms are frequent with the arid and desert-style terrains (9) (Figure 1).

The presence of raging rivers, sugarcane fields, hydroelectric and agricultural dams, and the optimum temperature and humidity have made Khuzestan a favorable habitat for the Bulinus truncates, snail which is the intermediate host for S. haematobium. Surprisingly, Khuzestan is the only province in Iran in which schistosomiasis has been reported. In 1949 a comprehensive survey by the institute of public health in Tehran University showed that schistosomiasis can only be found in Khuzestan province (8). Of different species of schistosomes, only S. haematobium has been reported in Iran; so, only urinary syndrome has been diagnosed in this area.

\subsection{Burden of the Disease in the Past}

Attempts to control urinary schistosomiasis in Iran were started in 1959 and a large amount of baseline data was collected before experimental and large-scale control operations were introduced (8). These investigations demonstrated that the infection was limited to a few foci in Khuzestan province and the number of infected persons was estimated to be $25000-30000$. More than 400 villages were observed to be infected in six main foci in Dezful, north of Ahwaz, Shushtar, Bidruyeh, Khoramshahr, and Dasht-e-Azadegan (formerly named Dasht-e-Mishan) areas. In some areas, more than $90 \%$ of people were infected. About $64 \%$ of cases were male and about $50 \%$ were in the age group of 11 - 15 years old. Most of these infections were observed in spring, early summer and autumn, when the number and activity of the intermediate snail is the maximum (10-12). In 1968, a WHO-assisted schistosomiasis control project was stablished. Control measures were started in endemic areas using a combination of chemotherapy with various antischistosomal drugs and snail control, including sanitary measures, environmental improvement, and focal mollusciciding of snail habitats (8). Selective population chemotherapy and mollusciciding were carried out throughout the year, but the main activities were concentrated during spring and autumn. During this program, more than 1.5 million urine samples were taken from suspected patients and thousands of cases were treated. All the suspected patients with hematuria and/or bladder irritability symptoms were screened by examination of urine. Urine samples were collected at 10:00 am and sediments of them were examined for S. haematobium ova under a stereoscopic microscope. Infected subjects were then treated with $30 \mathrm{mg}$ of niridazole per $\mathrm{kg}$ of body weight daily for four consecutive days. Because of the side-effect of this drug and its poor tolerability, it was replaced by praziquantel. Fallow-up urine samples also were taken from the patients to confirm their cure. Any patient had his own folder and all the epidemiological data, laboratory findings, prescribed drugs, and the outcomes of the therapy were recorded. The prevalence of infection in rural areas was also assessed annually by examination of urine samples. Active case finding was also performed by examination of urine samples from children less than 15 years old from several villages in various parts of the endemic area with known cases of S. haematobium infection or in areas in which the intermediate snail was detected. All the children found negative in the initial survey were re-examined at yearly intervals to determine the incidence of the infection. In addition, reports from physicians' offices and hospitals were recorded and treated. Beside these efforts, education programs were conducted for people living in endemic areas to break the transmission cycle through reduced human-water contact and diminished environmental contamination with excreta. Projects for improvements in water supply and sanitation, waterrelated infrastructures and water resources development were also conducted $(8,13)$. The snail intermediate host of S. haematobium in Iran is B. truncatus, which also acts as the snail host for S. bovis and Paramphistomum microbothrium, both in ruminants. Bulinus snails were found in various habitats such as borrow-pits, swamps, and canals. The peak snail population was seen in May-July and OctoberDecember. Most of the potential habitats in the area were surveyed twice a year using a wire-mesh net, and the infested habitats were treated with a niclosamide molluscicide (Table 1). Meanwhile, various sanitary measures were instituted, such as draining of the huge swamps, improvement of the canals, and provision of a standard latrine and fresh water supply for each family (8). The success of the program was assessed by monitoring the incidence of infection among school children in various villages. During this program, the overall incidence of infection decreased from 3.5\% in 1970 to 0 in 1977. However, a few newly infected cases were detected in 1978 and 1979 among school children under 15 years of age in several villages around Dezful (0.1\% in 1978 and 0.7\% in 1979) (8). Table 2 shows the 


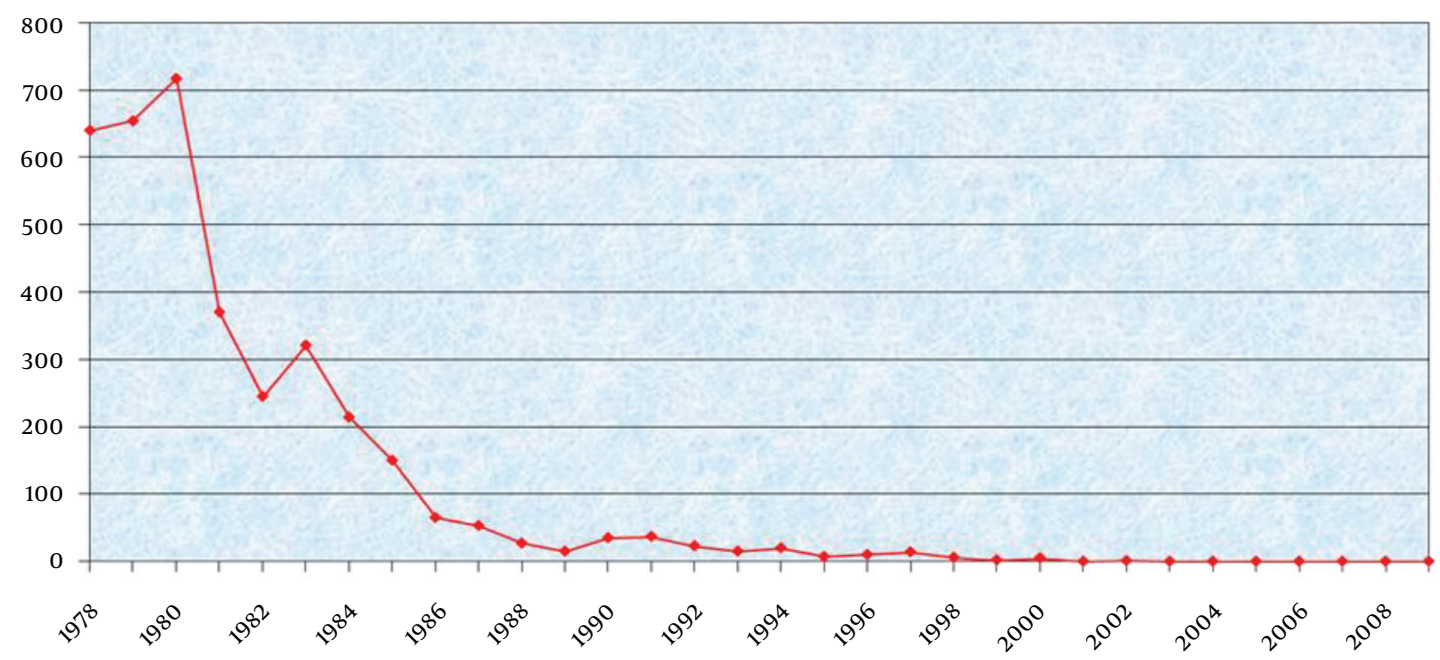

Figure 1. Trend of Schistosomiasis Cases in Iran (1978 - 2010)

annual prevalence of S. haematobium infection in Khuzestan, 1970 - 79 (3). From 1981 to 1990, 761 cases of S. haematobium were reported, and from 1991 to 2000 , only 99 cases were observed (8). In 2001, only one case was reported from west of Ahvaz among more than 40000 urine samples examined, and this was the last case of schistosomiasis in Khuzestan and of course in Iran (14). In the past decade, in over thousands of urine samples taken, no eggs were detected and no patient was diagnosed (Figure 2). Despite the absence of schistosomiasis in Khuzestan, urine sampling from suspected cases, investigation of previously infected villages, and monitoring of the natural habitats for B. truncatus snail is currently continuing (Figure 3). From multiple foci in the past, at present, only nine foci are diagnosed as natural habitats for $B$. truncatus in Khuzestan, two foci in Andimeshk and seven foci in Dezful districts (Table 3).

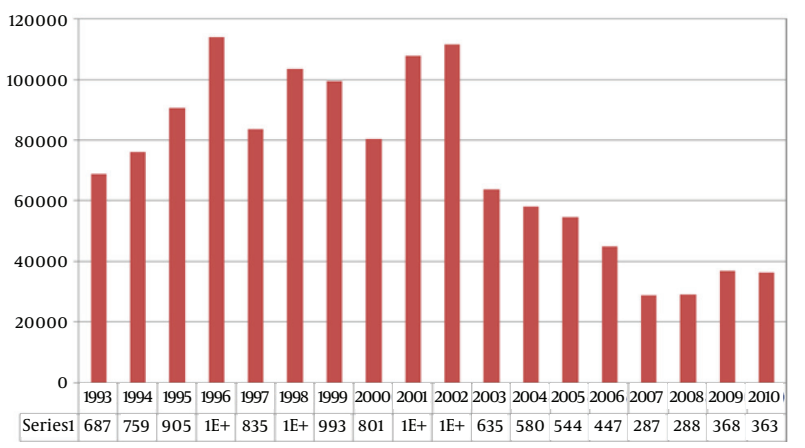

Figure 2. Annual Number of Urine Samples for Schistosoma haematobium Surveillance in Khuzestan Province of Iran (1993-2010)

\subsection{New Strategy for the Elimination Phase}

The national guideline for schistosomiasis control was revised in 2006 and schistosomiasis control program was changed from its previous vertical form to an integrated form in the present health service delivery system (15). The health service delivery system in Islamic republic of Iran is characterized by its tiered design with parallel structure in urban and rural areas (Figure 4). The first tier of delivery encompasses primary health care (PHC) and comprises two levels of care. The first level includes rural health houses with a defined served population of 1400 individuals. Health houses are staffed by Behvarzes (allied health workers). Behvarzes are selected and then trained in Behvarz training centers (BTC) for two years, which consists of training and service in health houses. This level also includes rural health centers which are staffed by a physician and a health worker. These centers supervise a number of health houses with a defined service population of 6000 to 10000 individuals. The second level of the PHC tier is the district health center which undertakes planning and supervising functions. In urban areas, there is a similar structure that includes health posts, serving a population of 12000 individuals, then urban health centers (with a defined service population of 40000 to 60000 individuals) and district health centers $(14,15)$. In Khuzestan, there are 26 district health centers, 79 urban health centers, 121 rural health centers, 62 urban-rural health centers, 209 health posts, and 859 health houses (13). By using the capacity of such health network, primary health care is distributed in all over the country from the most peripheral villages (health house) to the big cities. Surveillance programs for many diseases is conducted through this network and of 
Table 1. Proportion of Bulinus-Infested Habitats and Details of Mollusciciding in Khuzestan, 1970 - 79

\begin{tabular}{|c|c|c|c|c|}
\hline Year & No. of Habitats Tested & Positive Habitats, No. (\%) & No. of Habitats Treated & Amount of Niclosamid Used, $\mathrm{kg}$ \\
\hline 1970 & 5080 & $322(6.3)$ & 190 & 942.5 \\
\hline 1971 & 3148 & $318(10.1)$ & 199 & 1775 \\
\hline 1972 & 3164 & $336(10.6)$ & 105 & 1193 \\
\hline 1973 & 5946 & $408(6.9)$ & 110 & 2362 \\
\hline 1974 & 8420 & $412(4.9)$ & 122 & 3034.5 \\
\hline 1975 & 11520 & $325(2.8)$ & 189 & 1125.5 \\
\hline 1976 & 12727 & $288(2.3)$ & 94 & 3505 \\
\hline 1977 & 9952 & $244(2.5)$ & 25 & 869.5 \\
\hline 1978 & 12184 & $242(2.0)$ & 44 & 702 \\
\hline 1979 & 14374 & $348(2.4)$ & 104 & 1372 \\
\hline
\end{tabular}

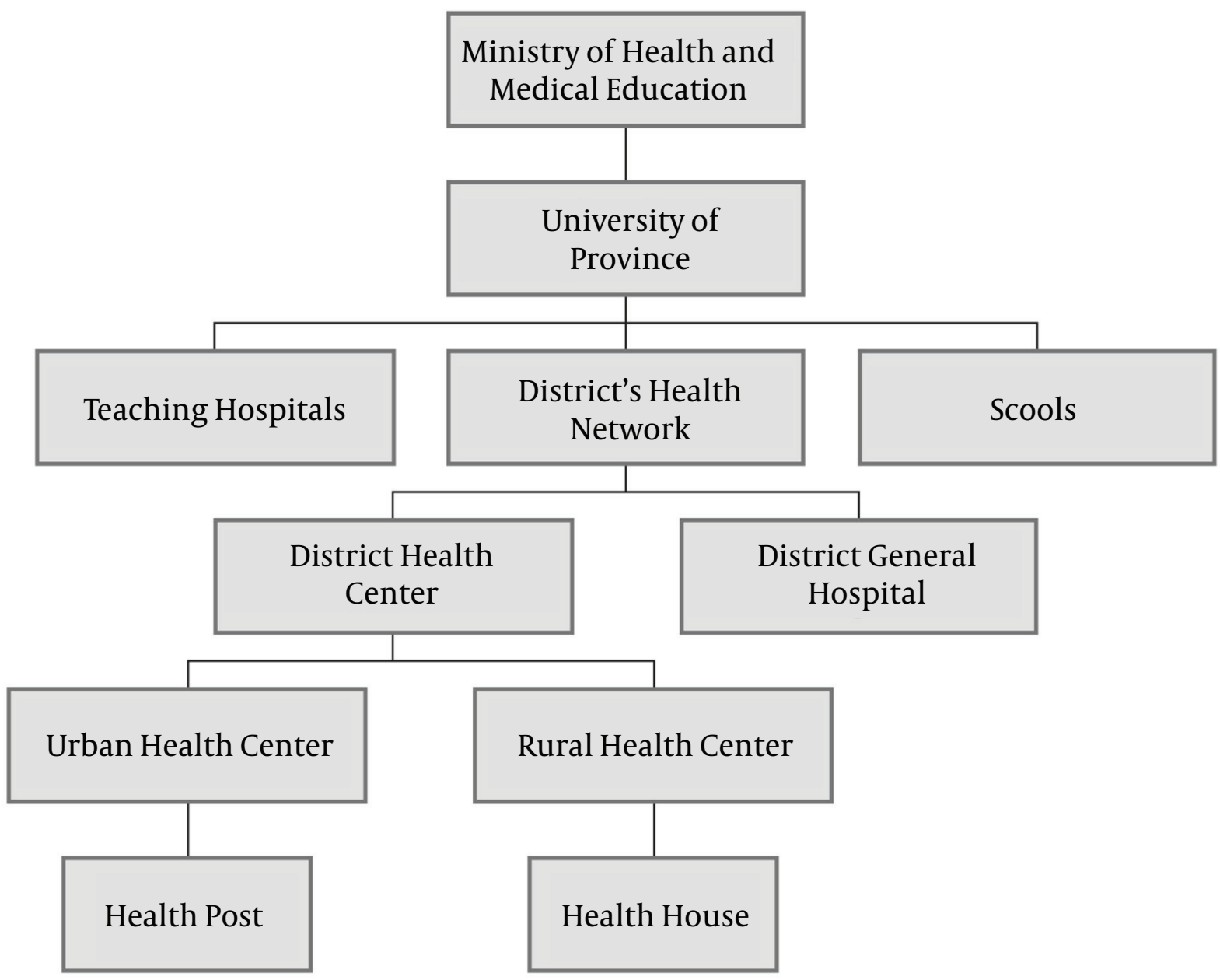

Figure 3. Health System Network in Iran 
Table 2. Annual Prevalence of Schistosoma haematobium in Khuzestan, 1970 - 79

\begin{tabular}{|cccc}
\hline Year & $\begin{array}{c}\text { No. of Urine Samples } \\
\text { Examined }\end{array}$ & No. Positive & Infection Rate, \% \\
\hline $\mathbf{1 9 7 0}$ & 21878 & 1822 & 8.3 \\
\hline $\mathbf{1 9 7 1}$ & 19919 & 1060 & 5.3 \\
\hline $\mathbf{1 9 7 2}$ & 30421 & 1160 & 3.8 \\
\hline $\mathbf{1 9 7 3}$ & 60534 & 1899 & 3.1 \\
\hline $\mathbf{1 9 7 4}$ & 71300 & 1206 & 1.7 \\
\hline $\mathbf{1 9 7 5}$ & 65370 & 832 & 1.3 \\
\hline $\mathbf{1 9 7 6}$ & 56057 & 655 & 1.2 \\
\hline $\mathbf{1 9 7 7}$ & 34973 & 258 & 0.74 \\
\hline $\mathbf{1 9 7 8}$ & 90046 & 801 & 0.89 \\
\hline $\mathbf{1 9 7 9}$ & 91022 & 674 & 0.74 \\
\hline
\end{tabular}

Table 3. Number of Natural Habitats for Bulinus truncatus in Khuzestan

\begin{tabular}{lc}
\hline Year & Number \\
\hline Before 1981 & 207 \\
\hline 1981 - 1990 & 265 \\
\hline 1991 - 2000 & 869 \\
\hline 2001 - 2011 & 272 \\
\hline 2012 & 9 \\
\hline Total & 1622 \\
\hline
\end{tabular}

course it helps the schistosomiasis control program in the elimination phase for more accurate case finding and early warning, if a suspected case is reported.

The new surveillance system for schistosomiasis in the elimination phase has recently been published by center for disease control of ministry of health of Iran. It is based on active and passive case finding in previously infected areas, monitoring of snails population, distribution and activity by the use of geographic information system (GIS) and global positioning systems (GPS) and implementation of appropriate control measures, education including community education and training courses for health care workers, performing practical researches by the use of classic, serologic and molecular methods, and also multicenter researches and increasing of national, regional and international cooperation.

\subsection{Challenges in the Elimination Phase}

The most important challenges for maintaining the elimination status includes development of irrigation and agricultural project such as sugarcane in Khuzestan province, which may lead to the increase of snail population. As schistosomiasis is eliminated and new medical students are not familiar with the disease, the knowledge of physicians and health workers about schistosomiasis is decreasing. The presence of active foci of schistosomiasis in southern regions of Iraq and increased trans-border traffic as well as travelling of people to visit shrine sites between two countries are other challenges in the elimination phase.

\section{Conclusions}

By using a national program for schistosomiasis control based on active and passive case finding, treatment of the patients (16), population education, conducting training courses for health workers, snail control programs including sanitary measures, environmental improvement, focal mollusciciding of snail habitats, and improvement of water supply for people living in endemic areas, schistosomiasis is eliminated from Iran and this can be a successful model for countries suffering from this disease.

\section{Footnote}

Authors' Contribution: Seyed Mohammad Alavi and Shokrollah Salmanzadeh had equal roles in writing of the paper. 


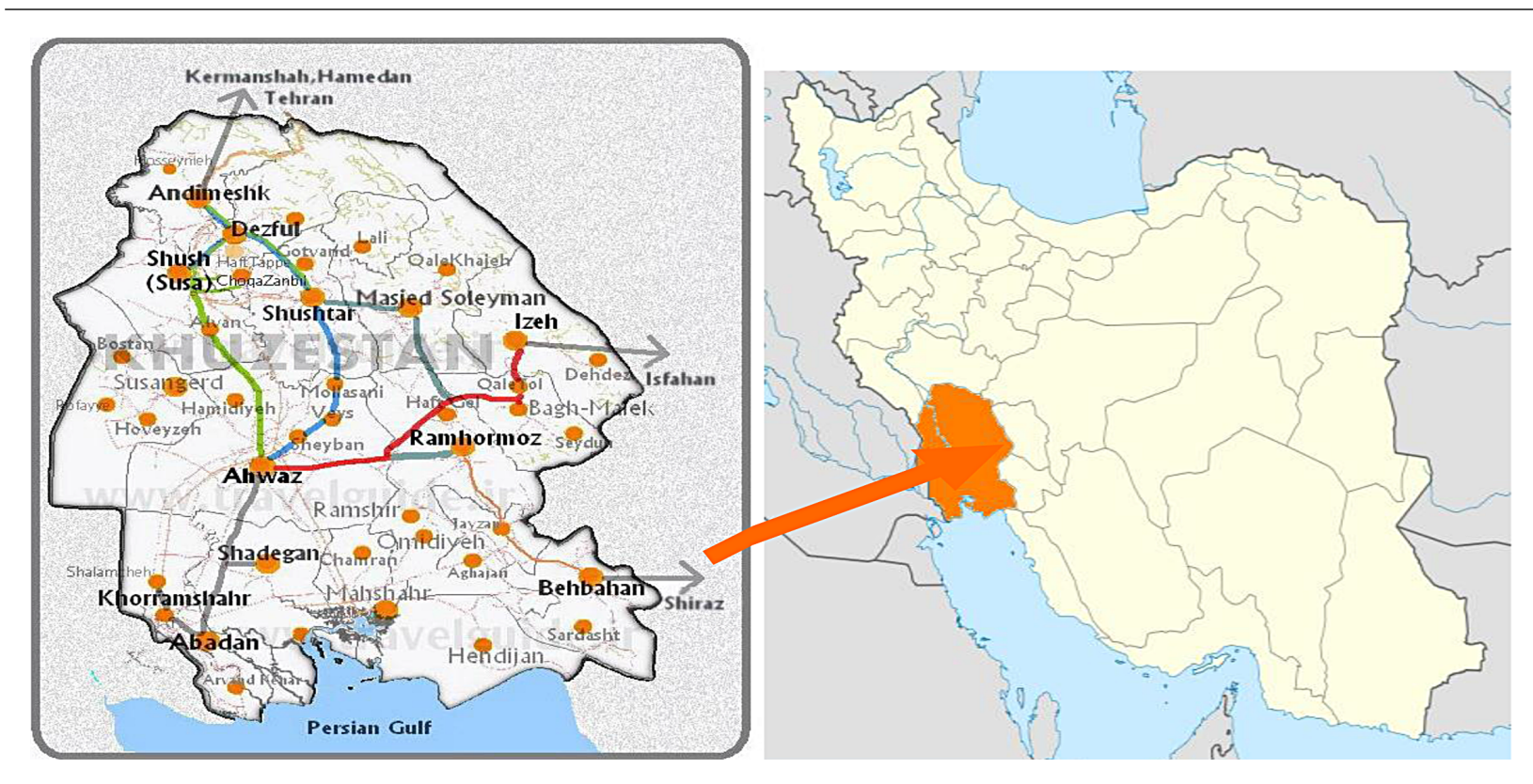

Figure 4. Khoozestan Province

\section{References}

1. Chitsulo L, Engels D, Montresor A, Savioli L. The global status of schistosomiasis and its control. Acta Trop. 2000;77(1):41-51. [PubMed: 10996119].

2. Chu TB, Liao CW, D'Lamini P, Chang PW, Chiu WT, Du WY, et al. Prevalence of Schistosoma haematobium infection among inhabitants of Lowveld, Swaziland, an endemic area for the disease. Trop Biomed. 2010;27(2):337-42. [PubMed: 20962734].

3. Engels D, Chitsulo L, Montresor A, Savioli L. The global epidemiological situation of schistosomiasis and new approaches to control and research. Acta Trop. 2002;82(2):139-46. [PubMed: 12020886].

4. Hotez PJ, Kamath A. Neglected tropical diseases in sub-saharan Africa: review of their prevalence, distribution, and disease burden. PLoS Negl Trop Dis. 2009;3(8):ee412. doi: 10.1371/journal.pntd.0000412. [PubMed: 19707588].

5. Steinmann P, Keiser J, Bos R, Tanner M, Utzinger J. Schistosomiasis and water resources development: systematic review, meta-analysis, and estimates of people at risk. Lancet Infect Dis. 2006;6(7):411-25. doi: 10.1016/s1473-3099(06)70521-7. [PubMed: 16790382].

6. Ketabchi A, Moshtaghi-Kashanian G. Urinary schistosomiasis with simultaneous bladder squamous cell carcinoma and transitional cell carcinoma. Iran J Parasitol. 2012;7(3):96-8. [PubMed: 23109968].

7. World Health Organization . Muscat, Oman: WHO; 2007. WHO report, Inter-country Meeting On Strategies to Eliminate Schistosomi- asis from the Eastern Mediterranean Region; .

8. Massoud J, Arfaa F, Farahmandian I, Ardalan A, Mansoorian A. Progress in the national schistosomiasis control programme of Iran. Bull World Health Organ. 1982;60(4):577-82. [PubMed: 6982780].

9. wikipedia . Khuzestan province Available from: http://en.wikipedia. org/wiki/Khuzestan_Province.

10. Gaud J. Observationssur la biologie de Bulinus truncatus au Khuzestan (Iran). Ann parasitol hum comp. 1962;37:235-75.

11. Arfaa F, Bijan H, Farahmandian I. Present status of urinary bilharziasis in Iran. Trans Royal Soc Trop Med Hyg. 1967;61(3):358-67.

12. Chu KY, Massoud J, Arfaa F. Distribution and ecology of Bulinus truncatus in Khuzestan, Iran. Bull World Health Organ. 1968;39(4):607-37. [PubMed: 5304436].

13. Mombeni H, Kheradmand A. Schistosomahaematobium Control in Khoozestan Province in Iran: Prosperities and Failures. Med J Islamic Repub Iran (MJIRI). 2005;19(1):19-22.

14. National committee of schistosomiasis MOHAME. Tehran, Iran: Ministry of health and medical education; 2006. National guideline for schistosomiasis control; .

15. Mehrdad R. Health System in Iran. JMAJ. 2009;52(1):69-73.

16. Silva IM, Thiengo R, Conceicao MJ, Rey L, Lenzi HL, Pereira Filho E, et al. Therapeutic failure of praziquantel in the treatment of Schistosoma haematobium infection in Brazilians returning from Africa. Mem Inst Oswaldo Cruz. 2005;100(4):445-9. doi: /S0074-02762005000400018. [PubMed: 16113896]. 